\title{
RESEARCH HIGHLIGHT Probing the motivational circuitry of binge eating
}

\author{
David A. Martin ${ }^{1}$ and Donna J. Calu ${ }^{1,2}$ \\ Neuropsychopharmacology (2020) 45:577-578; https://doi.org/10.1038/s41386-019-0568-4
}

The steady increase in obesity rates over the past four decades necessitates preclinical models of "food addiction" that accurately capitulate the psychological hallmarks of overeating. Neurobiological inquiry is critical to understand the motivational and hormonal dysregulation that occurs with overconsumption of calorically dense and palatable foods. In this issue of Neuropsychopharmacology, Spierling et al. carefully considered diagnostic features of food addiction to develop and validate motivational constructs in a variant of the intermittent access to palatable food-seeking model. After identifying a subset of rats that develop compulsive-like foodseeking behavior, the authors studied the associated hormonal profiles and corticostriatal pathway involvement [1].

By extending their previously developed model [2,3], Spierling et al. draw from drug self-administration literature, aiming to examine the effect of brief versus long intermittent access to highly palatable diet exposure on the emergence of compulsive-like motivation in female rats. Classic compulsivity measures include persistence of drug seeking when the drug is unavailable (responding during no-drug periods), high motivation (increased progressive-ratio responding), and continued responding despite harmful consequences (shockpunished responding) [4]. Here, Spierling et al. identified the compulsive-like behaviors arising in a subset of rats exposed intermittently to highly palatable food [1]. The authors used four experimental groups, including a continuous access to chow-only group, a continuous access to the preferred, high-sucrose, nutritionally complete diet (palatable), a brief-access intermittent group with $30 \mathrm{~min}$ of access to the palatable diet every $48 \mathrm{~h}$, and a long-access intermittent group with $24 \mathrm{~h}$ of access to the palatable diet every $48 \mathrm{~h}$.

Consistent with prior studies $[3,5-7]$, rats consumed the palatable diet at high levels when it was intermittently available and restricted chow consumption during periods when only chow was available. On days that investigators probed motivation to lever press for food, intermittent palatable rats gained access to palatable food at the start of the self-administration session, while continuous chow and palatable groups had access to their respective diets in the hours surrounding the instrumental session. Keeping with their prior study [2], the authors provided the intermittent groups with the same palatable pellets for the homecage- palatable diet as they used for the instrumental reinforcer, which prevented the potential for negative-contrast effects in the operant context [8,9].

Compared with continuous-access groups lever pressing for their respective diets, the two intermittent groups exhibited uniformly higher fixed-ratio (FR) responding, timeout responding, and progressive ratio (PR) responding. The voluntary chow restriction in intermittent groups may have contributed to their greater levels of palatable pellet self-administration compared with continuous- access groups self-administering their respective diets. Collectively, the intermittent-access groups exhibited increased motivation for the palatable food. However, when testing for continued responding despite adverse consequences (every third reinforcer during the FR schedule produced a shock), only a subset of punishment-resistant rats from intermittent-access groups continued to press for the palatable diet. Thus, individual differences coupled with scheduling effects combined to produce a compulsive-like phenotype in a subset of rats, akin to previous results using cocaine as the reinforcer [4].

Hormonal profiles indicative of high leptin and low ghrelin correlated with motivational measures in a subset of intermittent palatable-access rats showing the greatest PR responding. Spierling et al. also present evidence that compared with continuous- access rats, intermittent-access rats exhibited heightened aggression in a bottle brush test conducted immediately before palatable diet access, raising the possibility that intermittent access to palatable food promotes a negative affective state during periods of forced abstinence from palatable diets. Having established differences in multiple measures of motivation between groups, Spierling et al. are well suited to probe individual differences in motivational constructs and the neurobiological processes driving high levels of palatable food seeking.

The authors focused on anterior insular cortex (AIC) to nucleus accumbens (Acb) projections for their first putative target underlying the compulsive-like behaviors observed in their model. This pathway is implicated in driving reward (ethanol) seeking despite punishment [10], a measure in this study that distinguished punishment-sensitive and resistant rats exclusively in intermittentaccess groups. By using an optogenetic inhibition approach, they investigated the effect of inhibiting AIC terminals in Acb during FR and PR schedules of reinforcement, and FR under punishment in three groups of interest. Intermittent-access rats were divided into high and low responding groups based on their PR responding relative to an ad libitum group (combined continuous chow and palatable rats). Inhibition of AIC-Acb projections reduced PR responding only in intermittent high-responding rats, suggesting that this pathway mediates heightened motivation in this subset of rats. However, the optogenetic manipulation in intermittent high-responding rats had marginally potentiating effects on FR responding under purely rewarding conditions, and increased FR responding under punishment conditions-in contrast with a prior study in which photoinhibition of AIC-Acb reduced alcohol seeking under punishment conditions [10]. The dissociable effects of inhibiting AIC-Acb reveal distinct contributions of this neurocircuitry in modulating motivated behavior under different reinforcement schedules and appetitive/aversive conflict conditions and underscore the complexity of constructs supporting motivation for palatable food seeking in this model.

Collectively, the findings of the Spierling study set the stage for further behavioral and circuit inquiry testing the contributions of

\footnotetext{
${ }^{1}$ Department of Anatomy and Neurobiology, University of Maryland School of Medicine, Baltimore, MD, USA and ${ }^{2}$ Program in Neuroscience, University of Maryland School of Medicine, Baltimore, MD, USA

Correspondence: Donna J. Calu (Dcalu@som.umaryland.edu)
}

Received: 30 October 2019 Accepted: 3 November 2019

Published online: 18 November 2019 
diet scheduling and individual differences to the compulsive-like behaviors observed in this model. These preclinical efforts aimed at modeling the psychological hallmarks of binge eating may help us to translate the relevant contributing factors to "food addiction"-related disorders in humans, including obesity, bingeeating disorder, and bulimia nervosa.

\section{FUNDING AND DISCLOSURE}

This work was supported by a McKnight Memory and Cognitive Disorders Award (DJC), a NARSAD Young Investigator Grant \#24950 (DJC), NIDA grant R01DA043533 (DJC), and the Department of Anatomy and Neurobiology at the University of Maryland, School of Medicine. The authors declare no competing interests.

\section{AUTHOR CONTRIBUTIONS}

Both DAM and DJC contributed to conception of highlight, drafting, and revising of the article and have given final approval of the version to be published.

\section{ADDITIONAL INFORMATION}

Publisher's note Springer Nature remains neutral with regard to jurisdictional claims in published maps and institutional affiliations.

\section{REFERENCES}

1. Spierling S, Guglielmo G, Kirson D, et al. Insula to ventral striatal projections mediate compulsive eating produced by intermittent access to palatable food. Neuropsychopharmacol. 2019. https://doi.org/10.1038/s41386-019-0538-x.

2. Spierling SR, et al. Physiol Behav. 2018;192:3-16.

3. Kreisler AD, Garcia MG, Spierling SR, Hui BE, Zorrilla EP. Physiol Behav. 2017;177:305-16.

4. Deroche-Gamonet V, Belin D, Piazza PV. Science. 2004;305:1014-7.

5. Chen YW, Fiscella KA, Bacharach SZ, Calu DJ. PLoS ONE. 2014;9:e102213.

6. Cottone P, Sabino V, Steardo L, Zorrilla EP. Am J Physiol Regul Integr Comp Physiol. 2008;295:R1066-76.

7. Johnson PM, Kenny PJ. Nat Neurosci. 2010;13:635-41.

8. Cottone P, Sabino V, Steardo L, Zorrilla EP. Neuropsychopharmacology. 2008;33:524-35.

9. Crespi LP. Am J Psychol. 1942;55:467-517.

10. Seif T, et al. Nat Neurosci. 2013;16:1094-100. 